Interarm differences in systolic blood pressure and mortality among US army veterans aetiological associations and risk prediction in the Vietnam experience study

White, James; Mortensen, Laust Hvas; Kivimäki, Mika; Gale, Catharine R; Batty, G David

Published in:

European Journal of Preventive Cardiology

DOI:

$10.1177 / 2047487313496193$

Publication date:

2014

Document version

Publisher's PDF, also known as Version of record

Document license:

CC BY-NC

Citation for published version (APA):

White, J., Mortensen, L. H., Kivimäki, M., Gale, C. R., \& Batty, G. D. (2014). Interarm differences in systolic blood pressure and mortality among US army veterans: aetiological associations and risk prediction in the Vietnam experience study. European Journal of Preventive Cardiology, 21(11), 1394-400.

https://doi.org/10.1177/2047487313496193 


\title{
Interarm differences in systolic blood pressure and mortality among US army veterans: aetiological associations and risk prediction in the Vietnam experience study
}

European Journal of Preventive Cardiology

20I4, Vol. 2I(II) I394-1400 (C) The European Society of Cardiology 2013

Reprints and permissions: sagepub.co.uk/journalsPermissions.nav DOI: I0.II77/20474873|3496| 93 ejpc.sagepub.com

(s)SAGE

\author{
James White', Laust H Mortensen², Mika Kivimäki ${ }^{3}$, \\ Catharine R Gale ${ }^{4}$ and G David Batty ${ }^{3}$
}

\begin{abstract}
Background: Differences between the arms in systolic blood pressure (SBP) of $\geq 10 \mathrm{mmHg}$ have been associated with an increased risk of mortality in patients with hypertensive and chronic renal disease. For the first time, we examined these relationships in a non-clinical population.

Design: Cohort study.

Methods: Participants were 4419 men (mean age 38.37 years) from the Vietnam Experience Study. Bilateral SBP and diastolic BP (DBP), serum lipids, fasting glucose, erythrocyte sedimentation rate, metabolic syndrome, and ankle brachial index were assessed in 1986.

Results: Ten per cent of men had an interarm difference of $\geq 10$ and $2.4 \%$ of $\geq 15 \mathrm{mmHg}$. A 15 -year follow-up period gave rise to 246 deaths ( 64 from cardiovascular disease, CVD). Interarm differences of $\geq 10 \mathrm{mmHg}$ were associated with an elevated risk of all-cause mortality (hazard ratio, HR, I.49, 95\% confidence interval, $\mathrm{Cl}, \mathrm{I} .04-2.14$ ) and CVD mortality (HR 1.93, 95\% Cl 1.0I-3.69). After adjusting for SBP, DBP, lipids, fasting glucose, and erythrocyte sedimentation rate, associations between interarm differences of $\geq 10 \mathrm{mmHg}$ and all-cause mortality (HR I.35, 95\% Cl 0.94-I.95) and CVD mortality $(1.62,95 \% \mathrm{Cl} 0.84-3.14)$ were significantly attenuated.

Conclusions: In this non-clinical cohort study, interarm differences in SBP were not associated with mortality after accounting for traditional CVD risk factors. Interarm differences might not be valuable as an additional risk factor for mortality in populations with a low risk of CVD.
\end{abstract}

\section{Keywords}

Epidemiology, hypertension, risk factors

Received 6 February 2013; accepted 9 June 2013

\section{Introduction}

A marked difference in systolic blood pressure (SBP) between the arms have been linked to subclavian stenosis, ${ }^{1,2}$ atherosclerotic plaque, ${ }^{3}$ and are most commonly observed in patients with hypertension, ${ }^{4}$ diabetes, ${ }^{5}$ and chronic renal disease, ${ }^{1}$ suggesting interarm differences are a marker of peripheral vascular disease. ${ }^{6}$ The presence of an interarm difference has been linked to the delayed diagnosis ${ }^{7}$ and poor control of hypertension, ${ }^{8}$ and as such are recommended in screening guidelines on the assessment of hypertension. ${ }^{9,10}$ Despite these associations, few cohorts record information on systolic blood pressure (SBP) in both arms and as a result associations with mortality are unclear. A small number of

\footnotetext{
'Cardiff University, Cardiff, UK

${ }^{2}$ University of Copenhagen, Copenhagen, The Netherlands

${ }^{3}$ University College London, London, UK

${ }^{4}$ University of Southampton, Southampton, UK

\section{Corresponding author:}

James White, School of Medicine, Cardiff University, 4th Floor Neuadd Meirionnydd, Heath Park, Cardiff, CFI4 4YS, UK.

Email: whitejl I@cf.ac.uk
} 
studies have shown differences of $\geq 10 \mathrm{mmHg}$ between the arms in SBP are associated with an increased risk for all-cause $e^{4,6}$ and cardiovascular disease (CVD) mortality in high risk patients (e.g. hypertension, ${ }^{4,11}$ chronic renal disease ${ }^{1}$ ). However, owing to a paucity of data, the extent to which these results are apparent in nonclinical groups is unclear.

Accordingly, the aim of the present study was to examine whether interarm differences in SBP are associated with all-cause and CVD mortality over a 15 -year follow-up period in a cohort of middle-aged men. In addition to examining the link, if any, between interarm differences in blood pressure and mortality, we also test whether incorporating information on interarm differences into an established risk score for CVD (the Framingham risk algorithm) ${ }^{12}$ will improve its predictive capacity for all-cause and CVD mortality.

\section{Methods}

\section{Study design and participants}

Participants were drawn from the Vietnam Experience Study. The Vietnam Experience Study is a prospective cohort of US army veterans that was established in 1983 to compare the health of men who participated in the Vietnam war against those who did not. ${ }^{13}$ Briefly, on 31 December 1983, 18,313 men were drawn randomly from 5 million records of men who served in Vietnam and elsewhere. Of those men who were traced, 15,288 (85.6\% response) participated in a telephone survey in 1985. A random sample was taken of telephone survey respondents in 1986 and 4462 (69.3\% of those invited) attended a 3-day medical examination. Ethical approval for the study protocol was given by the US Office for Technology Assessment, the Department of Health and Human Sciences Advisory Committee, the Agent Orange Working Group Science Panel, and a review panel from the US Centers for Disease Control.

\section{Assessment of interarm differences in blood pressure}

Blood pressure was assessed after participants were seated for at least 2 minutes, with a standard mercury sphygmomanometer. Research nurses were instructed to ensure each arm was supported during measurements, free of clothing, and only to take measurements when men appeared relaxed and comfortable. Measurements were made twice in both arms in an alternating sequence: right arm, left arm, right arm, left arm. Standard or large cuffs were used as appropriate. ${ }^{14}$ Interarm differences in SBP were similar for the first (mean $1.00 \mathrm{mmHg}$; $95 \%$ CI $0.76-1.21$ ) and second pair of assessments $(1.05 \mathrm{mmHg}, 95 \%$ CI $0.80-1.29)$.
Readings were averaged to obtain a mean SBP for each arm, which was then used to calculate an interarm difference (mean of right arm minus mean of left arm).

\section{Assessment of risk factors for CVD mortality}

Blood samples were taken in the morning after participants had fasted from 19:00 the previous day. Levels of triglycerides, cholesterol fractions, and urinary creatinine were ascertained using a Ektachem 700 AutoAnalyzer (Eastman Kodak, Rochester, New York). Serum glucose level was determined with an adaptation of the glucose oxidase-peroxidase-chromogen-coupled system for glucose determination in biological fluids. Erythrocyte sedimentation rate (ESR) was measured using the Westergen method. Height and weight were measured from which body mass index was calculated.

We defined the metabolic syndrome using a modified version of the Adult Treatment Panel III criteria (using body mass index $\geq 30 \mathrm{~kg} / \mathrm{m}^{2}$ instead of waist circumference, regarded by the WHO as an acceptable substitute). ${ }^{15,16}$ Diabetes status was defined as having a fasting plasma glucose $\geq 7.0 \mathrm{mmol} / 1$ and/or use of medication for diabetes. ${ }^{17}$

Hypertension was defined using the JNC 7 cut-off points for systolic/diastolic blood pressure $\geq 140$ / $\geq 90 \mathrm{mmHg}$, or use of antihypertensive medication. ${ }^{9}$ Men underwent a separate examination of the peripheral arterial system to calculate the ankle brachial index (ABI) using the Doppler technique. ABI for each leg was calculated by dividing the ankle systolic pressure by the higher of the right and left brachial systolic pressures obtained during this examination, and we used the lower of the left and right leg indices in the analysis, as an indicator of worse disease. Low ABI was defined as $\mathrm{ABI} \leq 0.9$. $^{18}$

Scores for the risk of coronary heart disease at 10 years were calculated using the Framingham equation with information on age, sex, total cholesterol levels, high-density lipoprotein (HDL) cholesterol levels, SBP, and smoking habits. ${ }^{12}$

\section{Ascertainment of all-cause and CVD mortality}

Information on deaths was collected for 15 years after the 1986 medical examination. Mortality was ascertained using databases supplied by the US army: the Veterans Administration (Beneficiary Identification and Records Locator Subsystem), the Social Security Administration, the Internal Revenue Service, and the National Center for Health Statistics (National Death Index). Events were defined as deaths from all-causes and those resulting from major CVD (ICD-9: 390-434, 436-448; ICD-10: I00-I78). A previous analysis found 
the standardized (for age, race, and calendar year) mortality ratios of Vietnam Experience Study participants were comparable to those of an equivalent US male population. ${ }^{19}$

\section{Statistical analysis}

We compared participants' characteristics using ANOVA, chi-squared, and Kruskall-Wallis tests according to interarm differences in SBP of $0-4.9$, 5-9.9, 10-14.9, and $\geq 15 \mathrm{mmHg}$. We examined the proportional hazards assumption graphically for each interarm difference in SBP with all-cause and CVD mortality and found no evidence for violation. We therefore used Weibull regression analysis to examine the association between interarm differences and mortality (for all causes and CVD). This model is a parametric form of the Cox proportional hazards model and accounts for the differing lengths of follow up among participants. This model also allows researchers to calculate the risk for mortality during a fixed period of time $(t)$ as $\mathrm{r}(t): 1-\exp \left(-\exp ^{(\log (t)-X \beta) / \sigma}\right)$, where $\mathrm{X}$ is the vector of risk factors, $\beta$ is the vector of coefficients, and $\sigma$ is the estimated scale parameter. This model has been used previously in the Framingham Heart Study and to describe the effect of adding new risk factors for CVD to the Framingham risk score. ${ }^{20}$

Interarm SBP difference was fitted as a continuous term (per $10 \mathrm{mmHg}$ ) and in separate models, using categories of $<5$ vs. $\geq 5 \mathrm{mmHg},<10$ vs. $\geq 10 \mathrm{mmHg}$, and $<15$ vs. $\geq 15 \mathrm{mmHg}$. Hazard ratios and accompanying $95 \%$ CIs were sequentially adjusted for a series of potential confounding factors previously associated with peripheral vascular disease ${ }^{6}$ and CVD mortality. ${ }^{21,22}$ Adjustments were made for: obesity, smoking status, units of alcohol per week, and ethnic group (model 2); model 2 plus mean SBP and DBP (from the four measurements of SBP and DBP), metabolic syndrome, triglyceride, HDL cholesterol, glucose, ESR, and creatinine (model 3); model 3 plus ABI $(\leq 0.9$ vs $>0.9$; model 4$)$; and model 4 plus the Framingham risk score (model 5). We modelled an interaction between interarm differences at each cutoff point and hypertension status to examine whether associations were different in hypertensive men, as significant associations have been found in hypertensive patients before. ${ }^{4,11}$

We also calculated the hazards for mortality using a Weibull model that included the Framingham risk score and interarm differences in SBP (at $\geq 5, \geq 10$, and $\geq 15 \mathrm{mmHg}$ ) and compared it to a model that only included the Framingham risk score. We examined discrimination across the two models by using Harell's c-index adjusted for optimism using 100 bootstrap repetitions; ${ }^{23}$ an overall (likelihood ratio chi-squared test) and penalized assessment of model fit (Akaike's Information Criterion, ${ }^{24}$ and the Bayes Information Criterion). ${ }^{25} \mathrm{We}$ chose these measures of discrimination rather than the net reclassification improvement index, ${ }^{26}$ as there was a low number of deaths in men with high Framingham risk scores.

We then estimated the effect on the hazard ratio of adjusting for a particular covariate using the following formula: [(HR adjusted for age and ethnicity -1$)-$ (HR adjusted for age and ethnicity plus covariate -1 )/ (HR adjusted for age and ethnicity -1$)] \times 100$. All analyses were performed with Stata version 11.0 using two-sided tests with a significance level of $p<0.05$.

\section{Results}

Of the 4462 men eligible for inclusion, 4419 (99.03\%) featured in the analytical sample. The 43 men who attended the medical examination but were excluded due to having missing values for some variables had a lower mean DBP (87.01 vs. $84.09 \mathrm{mmHg} ; p=0.04)$ and ESR ( 41.63 vs. $36.90 \mathrm{~mm} / \mathrm{h} ; p=0.03$ ) but were similar in other respects. Supplementary Table 1 (available online) shows that relative to study members not selected for the medical examination, men included in the analytical sample were more likely be obese (12.7 vs. $11.5 \%$ ), but generally differences in characteristics between men included and excluded were small.

Table 1 shows the characteristics of the 4419 participants. A total of $1667(37.7 \%)$ men had a mean interarm difference in SBP of $\geq 5 \mathrm{mmHg}, 435(9.8 \%)$ of $\geq 10 \mathrm{mmHg}, 107(2.4 \%)$ of $\geq 15 \mathrm{mmHg}$, and $24(0.5 \%)$ of $\geq 20 \mathrm{mmHg}$. The mean interarm difference in SBP was $1.03 \mathrm{mmHg}(95 \%$ CI $0.83-1.22)$, and was higher in the right arm. Interarm differences in SBP were positively associated with mean SBP $(0-4.9 \mathrm{mmHg}$ mean 122.7 vs. $\geq 15 \mathrm{mmHg}$ mean $128.25 ; p<0.001)$ and DBP $(0-4.9 \mathrm{mmHg}$ mean 83.79 vs. $\geq 15 \mathrm{mmHg}$ mean $85.71 ; p=0.01)$ and differences of 10 and $15 \mathrm{mmHg}$ were more common in men with hypertension $(p<0.001)$, an $\mathrm{ABI} \leq 0.9(p=0.01)$, and those who were obese $(p=0.04)$. There was, however, no evidence that interarm differences were associated with glucose levels or being defined as having diabetes or metabolic syndrome (Table 1).

A mean of 14.9 years of follow up (range 0.03-15.00) gave rise to $246(5.60 \%)$ deaths in total of which 64 $(26.01 \%)$ were due to CVD. Table 2 shows that in the unadjusted regression models, the hazard ratios for allcause mortality among men with an interarm difference in SBP of $>5,>10 \mathrm{~m}$ and $>15 \mathrm{mmHg}$ were $1.30(95 \%$ CI 1.01-1.67), 1.49 (95\% CI 1.04-2.14) and 1.37 (95\% CI $0.68-2.77)$ respectively, with stronger associations apparent for CVD mortality: for $>5 \mathrm{mmHg}$ : HR 1.66 , $95 \%$ CI $1.02-2.71$; >10 mmHg: HR $1.93,95 \%$ 
Table I. Characteristics of participants according to interarm differences in systolic blood pressure in the Vietnam Experience Study (1986)

\begin{tabular}{|c|c|c|c|c|c|}
\hline Variable & $\begin{array}{l}0-4.9 \mathrm{mmHg} \\
(n=2752)\end{array}$ & $\begin{array}{l}5-9.9 \mathrm{mmHg} \\
(n=1232)\end{array}$ & $\begin{array}{l}10-14.9 \mathrm{mmHg} \\
(n=328)\end{array}$ & $\begin{array}{l}\geq 15 \mathrm{mmHg} \\
(n=107)\end{array}$ & $P_{\text {trend }}$ \\
\hline Age (years) & $38.36 \pm 2.51$ & $38.27 \pm 2.49$ & $38.23 \pm 2.57$ & $38.65 \pm 2.67$ & 0.37 \\
\hline Systolic blood pressure $(\mathrm{mmHg})$ & $|22.27 \pm| \mid .57$ & $123.76 \pm 12.23$ & $124.27 \pm 12.92$ & $128.25 \pm 13.85$ & $<0.001$ \\
\hline Diastolic blood pressure $(\mathrm{mmHg})$ & $83.79 \pm 9.20$ & $84.40 \pm 9.36$ & $84.98 \pm 10.26$ & $85.71 \pm 10.86$ & 0.01 \\
\hline Glucose $(\mathrm{mmol} / \mathrm{l})$ & $5.22 \pm 0.93$ & $5.21 \pm 0.84$ & $5.34 \pm 1.47$ & $5.29 \pm 0.49$ & 0.14 \\
\hline Triglycerides (mg/dl) & I.0I $(0.70-\mid .5 \mathrm{I})$ & $1.01(0.69-1.46)$ & $1.08(0.76-1.57)$ & $1.06(0.75-1.59)$ & 0.08 \\
\hline HDL cholesterol (mg/dl) & $1.16 \pm 0.33$ & $1.15 \pm 0.30$ & $1.13 \pm 0.29$ & $1.10 \pm 0.31$ & 0.07 \\
\hline Erythrocyte sedimentation rate $(\mathrm{mm} / \mathrm{h})$ & $36.78 \pm 17.15$ & $36.7 I \pm 17.28$ & $37.38 \pm 17.13$ & $40.51 \pm 20.64$ & 0.16 \\
\hline Creatinine (mg/dl) & $\mathrm{I} . \mathrm{II} \pm 0.18$ & $\mathrm{I} . \mathrm{II} \pm 0.18$ & $1.10 \pm 0.18$ & $1.13 \pm 0.19$ & 0.40 \\
\hline Alcohol intake (units per week) & $2.00(0.00-9.00)$ & $2.00(0.00-8.00)$ & $2.00(0.00-9.00)$ & $1.00(0.00-8.00)$ & 0.24 \\
\hline \multicolumn{6}{|l|}{ Ethnic group } \\
\hline White & $81.9(2253)$ & 83.1 ( 1 024) & $78.4(257)$ & $83.2(89)$ & \\
\hline Black & II.8 (325) & II.5 (I42) & $12.8(42)$ & $7.5(8)$ & \\
\hline Other $^{a}$ & $6.3(174)$ & $5.4(66)$ & $8.8(29)$ & $9.3(10)$ & 0.15 \\
\hline Hypertension status ${ }^{b}$ & $27.9(768)$ & $28.6(352)$ & $36.0(118)$ & $47.7(5 \mathrm{I})$ & $<0.001$ \\
\hline Ankle brachial index $\leq 0.9$ & $3.3(92)$ & $3.7(46)$ & $4.3(14)$ & $9.3(10)$ & 0.01 \\
\hline Diabetes status $^{c}$ & $2.1(57)$ & $\mathrm{I} .7(2 \mathrm{I})$ & $2.4(8)$ & $0.9(1)$ & 0.67 \\
\hline Metabolic syndrome $^{d}$ & $15.8(435)$ & I4.5 (I79) & $18.3(60)$ & $19.6(21)$ & 0.24 \\
\hline Obesity $^{\mathrm{e}}$ & 12.1 (334) & $12.6(155)$ & $17.1(56)$ & $16.8(18)$ & 0.04 \\
\hline Current smoker & 44.1 (1 214) & $4 I .7(5 \mid 4)$ & $44.8(147)$ & $35.5(38)$ & 0.18 \\
\hline
\end{tabular}

Values are mean $\pm S D$, median (interquartile range), or\% $(n) ;{ }^{a}$ Other group $=$ Hispanics, Asians, Pacific Islanders, American Indians, and Alaskan Natives; ${ }^{b}$ Normotensive: systolic blood pressure $<140 \mathrm{mmHg}$ and diastolic blood pressure $<90 \mathrm{mmHg}$; hypertensive: systolic blood pressure $>140 \mathrm{mmHg}$ or diastolic blood pressure $8805 ; 90 \mathrm{mmHg}$ or on hypertension medication (the Seventh Report of the Joint National Committee on Prevention, Detection, Evaluation, and Treatment of High Blood Pressure criterion) or any hypertension medication; ${ }^{\mathrm{C}}$ Fasting blood glucose $\geq 7.0 \mathrm{mmol} / \mathrm{l}$ or on medication for diabetes; 'Modified version of the Adult Treatment Panel III diagnostic criteria using body mass index $\geq 30 \mathrm{~kg} / \mathrm{m}^{2}$ instead of waist

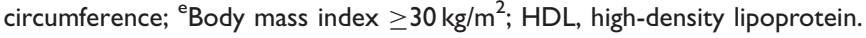

CI: $1.01-3.69 ;>15 \mathrm{mmHg}$ HR $2.00,95 \%$ CI $0.63-6.39$. When interarm difference was fitted as a continuous term (per $10 \mathrm{mmHg}$ ), there was positive association with both all-cause (HR 1.13, 95\% CI 1.01-1.27) and CVD mortality (HR $1.14,95 \%$ CI $0.92-1.40$ ) in the fully adjusted analysis (Supplementary Table 2).

Following adjustment for established risk factors for CVD, including the Framingham risk score, the adjusted hazard ratios (model 5) for interarm differences of $>10 \mathrm{mmHg}$ were significantly attenuated, with hazard ratios of $1.35(95 \% \mathrm{CI}=0.94-1.95)$ for all-cause and 1.62 (95\% CI 0.84-3.14) for CVD mortality. Supplementary Table 3, shows separate adjustments for factors associated with lifestyle (smoking status, units of alcohol per week, obesity status) at the baseline assessment explained little of the attenuating effect on these associations. In general, adjustment for hypertension status (all-cause mortality 6-25\%), metabolic syndrome (all-cause mortality 6-22\%), and the Framingham risk score (all-cause mortality 16$33 \%$ ) were associated with the largest percentage reduction in associations between interarm differences and mortality.

Harrell's C-indices indicated a moderate level of discrimination but estimates were not significantly different across the Framingham risk scores and Framingham plus interarm difference models. There was also little change in the penalized measures of model fit between the Framingham risk scores and Framingham plus interarm difference models (Supplementary Table 4). Interactions between interarm differences at 5, 10, and $15 \mathrm{mmHg}$ and hypertension status were also non-significant at each cut-off point for all-cause and CVD mortality ( $p>0.05$; data not tabulated).

\section{Discussion}

In a cohort of nearly 4500 men, an interarm difference in systolic blood pressure of $10 \mathrm{mmHg}$ or more was associated with an increased risk for CVD and all-cause mortality. This association was, however, significantly 
Table 2. Unadjusted and adjusted hazard ratios for all-cause and cardiovascular disease mortality at the cut-off points of $\geq 5, \geq 10$, and $\geq 15 \mathrm{mmHg}$ in systolic blood pressure $(n=4419)$

\begin{tabular}{|c|c|c|c|c|}
\hline \multirow[b]{2}{*}{ Model } & \multicolumn{2}{|c|}{ All deaths $(n=246)$} & \multicolumn{2}{|c|}{ Cardiovascular deaths $(n=64)$} \\
\hline & $\mathrm{HR}$ & $p$-value & $H R$ & $p$-value \\
\hline \multicolumn{5}{|l|}{ I } \\
\hline$\geq 5$ & $1.30(1.01-1.67)$ & 0.04 & I.66 (I.02-2.7I) & 0.04 \\
\hline$\geq 10$ & $1.49(1.04-2.14)$ & 0.03 & $1.93(1.01-3.69)$ & 0.04 \\
\hline$\geq 15$ & $1.37(0.68-2.77)$ & 0.38 & $2.00(0.63-6.39)$ & 0.24 \\
\hline \multicolumn{5}{|l|}{2} \\
\hline$\geq 5$ & $1.30(1.01-1.67)$ & 0.04 & I.67 (I.02-2.7I) & 0.04 \\
\hline$\geq 10$ & $1.43(1.00-2.06)$ & 0.05 & $1.84(0.96-3.53)$ & 0.07 \\
\hline$\geq 15$ & $1.53(0.75-3.09)$ & 0.24 & $2.13(0.67-6.82)$ & 0.20 \\
\hline \multicolumn{5}{|l|}{3} \\
\hline$\geq 5$ & $1.28(0.99-1.65)$ & 0.04 & $1.61(0.98-2.64)$ & 0.06 \\
\hline$\geq 10$ & $1.37(0.95-1.98)$ & 0.09 & $1.69(0.87-3.25)$ & 0.12 \\
\hline$\geq 15$ & $1.38(0.67-2.82)$ & 0.37 & $1.86(0.57-6.04)$ & 0.30 \\
\hline \multicolumn{5}{|l|}{4} \\
\hline$\geq 5$ & $1.28(0.99-1.65)$ & 0.06 & $1.61(0.98-2.64)$ & 0.06 \\
\hline$\geq 10$ & $1.35(0.94-1.95)$ & 0.11 & $1.67(0.87-3.23)$ & 0.12 \\
\hline$\geq 15$ & $1.28(0.62-2.62)$ & 0.50 & $1.85(0.57-6.04)$ & 0.30 \\
\hline \multicolumn{5}{|c|}{ 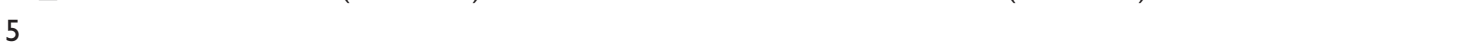 } \\
\hline$\geq 5$ & $1.28(0.99-1.65)$ & 0.06 & $1.56(0.95-2.56)$ & 0.08 \\
\hline$\geq 10$ & $1.35(0.94-1.95)$ & 0.11 & $1.62(0.84-3.14)$ & 0.15 \\
\hline$\geq 15$ & $1.28(0.62-2.63)$ & 0.50 & $1.62(0.49-5.34)$ & 0.43 \\
\hline
\end{tabular}

Values are hazard ratio (95\% confidence interval). Reference category, interarm difference in systolic blood pressure $<5,<10$, or $<15$, depending on the analysis; Model I, Unadjusted; Model 2, Model I + body mass index $\geq 30 \mathrm{~kg} / \mathrm{m}^{2}$, smoking status, alcohol intake (units per week), ethnic group; Model 3, Model $2+$ systolic blood pressure, diastolic blood pressure, metabolic syndrome, triglycerides, HDL cholesterol, glucose, erythrocyte sedimentation rate; Model 4. Model $3+$ ankle brachial index $\leq 0.9$; Model 5, Model $4+$ Framingham risk score.

attenuated after adjustment for established CVD risk factors. In regard to explanatory factors, smoking status, units of alcohol per week, and obesity played little or no part in the associations between interarm differences and mortality. ABI, a measure of peripheral artery disease, had a small but modest effect on the interarm difference-mortality association. Adjustment for hypertension, metabolic syndrome, and in particular Framingham risk scores at the baseline assessment had the strongest attenuating effects on the interarm difference - mortality association. Consistent with this finding, we found interarm differences did not improve upon the Framingham risk score in the prediction of mortality.

We identified one recent meta analysis ${ }^{6}$ and two cohort studies $^{4,27}$ not included in the meta-analysis that examined the association between interarm differences in SBP and all-cause and CVD mortality in patients with hypertension ${ }^{4}$ and renal disease. ${ }^{27} \mathrm{~A}$ positive association was reported in the meta analysis between all-cause mortality and an interarm difference of $10 \mathrm{mmHg}$ or more in SBP (pooled HR for two cohorts 1.90 (95\% CI $0.8-4.7){ }^{8,11}$ and two cohorts not included in this meta analysis reported results of a similar magnitude., ${ }^{4,27}$ One cohort of hypertensive patients reported larger hazards for CVD than allcause mortality after 5 (HR 2.8, 95\% CI $0.9-9.2)^{11}$ and 10 years of follow up (HR 4.2, 95\% CI $1.7-$ $10.8),{ }^{2-4,7,12}$ with a similar pattern reported in a cross-sectional analysis of patients undergoing haemodialysis. ${ }^{27}$ In contrast to these findings, we did not find an association between interarm differences in SBP and mortality. This inconsistency may in part be attributed to our deliberate selection of a low-risk cohort characterized by a comparatively low prevalence of hypertension, metabolic syndrome and other CVD risk factors. This resulted in a lower event rate and smaller interarm differences than have been reported in previous studies with hypertensive patients. ${ }^{4,11}$ In support of this assertion, we found a lower proportion of men with interarm differences at $10 \mathrm{mmHg}$ or more (13.1 vs. $24.0 \%)$ and $15 \mathrm{mmHg}$ or more (3.9 vs. $9.0 \%)^{4}$ and a lower proportion of deaths in the present cohort (all-cause mortality 5.4 vs. $25.7 \%)^{4}$ 
Despite these differences in sample characteristics, one study with hypertensive patients reported large hazard ratios for the interarm difference-mortality association even after adjusting for the Framingham risk score (difference of $10 \mathrm{mmHg}$ in SBP: HR 2.2; $95 \%$ CI $1.4-3.6){ }^{4}$ This suggests a mechanism by which interarm differences increase the risk for mortality which does not involve factors included in the Framingham risk score; and that this factor was not present in our cohort. Interarm differences have been conceptualized, and are associated with a measure of peripheral artery disease, the ABI. ${ }^{1,2}$ However, we found a low ABI indicative of peripheral artery disease did not explain associations between interarm differences and mortality. It is therefore possible that the cut-off point we used on the ABI lacks predictive validity that or interarm differences are indicative of another comorbidity in hypertensive patients not adequately captured by traditional CVD risk factors.

The strengths of this study are its size, being larger than previous studies on interarm difference, and tracking of participants over a 15 -year period. This allowed us to evaluate the prognostic value of interarm differences over a period in middle age where screening for hypertension typically starts. The non-clinical recruitment meant we could examine associations in a low-risk population for the first time and the comprehensive measurement of CVD risk factors allowed us to address concerns that interarm differences might not add anything over routine CVD risk factor assessment.

This study is not, however, without limitations. First, the sequential method of recording blood pressure we used may have produced larger interarm differences than simultaneous readings. ${ }^{28,29}$ Although, little difference has been found between these methods in estimates of the association of interarm differences with ABI, or all-cause mortality. ${ }^{6}$ Second, we did not have data on women, and all investigations to date have been in cohorts in middle to old age; , $^{1,8,11,27}$ thus, further testing is needed to confirm associations in women and different age groups. It is also worth noting that despite not adding to the prediction of mortality, assessment of blood pressure in both arms may have clinical value by increasing the sensitivity of diagnosis and management of hypertension through the use of measurements from the arm with the highest reading. Additional studies, ideally a controlled clinical trial, are needed to assess whether incorporating bi-lateral brachial assessments into screening for hypertension in primary care, would improve the identification and management of individuals at risk for a coronary heart disease event.

In conclusion, we found that interarm differences were associated with survival over a 15 -year period in men with a low risk of CVD, but that these associations were explained, in part, by traditional CVD risk factors. As these risk factors are already part of routine CVD risk screening, interarm differences may not offer anything over established risk prediction algorithms, such as the Framingham risk score, in the prediction of mortality in populations with a low risk of CVD.

\section{Funding}

This work was supported by the Centre for the Development and Evaluation of Complex Interventions for Public Health Improvement (by Dr White), a UKCRC Public Health Research: Centre of Excellence. Funding from the British Heart Foundation, Cancer Research UK, Economic and Social Research Council (ESRC RES-590-28-0005), Medical Research Council, the Welsh Assembly Government and the Wellcome Trust (WT087640MA), under the auspices of the UK Clinical Research Collaboration, is gratefully acknowledged. MK is supported by the MRC and by a professorial fellowship from the ESRC. CG and GDB are supported by the Centre for Cognitive Ageing and Cognitive Epidemiology, Department of Psychology, which is funded by the Biotechnology Sciences Research Council, the Engineering and Physical Sciences Research Council, the Economic and Social Research Council, the Medical Research Council and the University of Edinburgh as part of the cross-council Lifelong Health and Wellbeing initiative. The funding organizations played no role in the design and conduct of the study; collection, management, analysis and interpretation of the data; and preparation, review or approval of the manuscript. Dr. White had full access to all the data in the study and takes responsibility for the integrity of the data and accuracy of the analysis.

\section{Conflict of interest}

The authors declare that there is no conflict of interest.

\section{References}

1. Agarwal R, Bunaye Z and Bekele DM. Prognostic significance of between-arm blood pressure differences. Hypertension 2008; 51: 657-662.

2. Su H-M, Lin T-H, Hsu P-C, et al. Association of interarm systolic blood pressure difference with atherosclerosis and left ventricular hypertrophy. PLOS ONE 2012; 7: e41173.

3. HeTie C, Luo Y, Wen Z, et al. Development of the synchronous sphygmomanometer for four limbs - technical basics and medical practice. Chin J Biomed Eng 2002; 21 : 182-186.

4. Clark CE, Taylor RS, Shore AC, et al. The difference in blood pressure readings between arms and survival: primary care cohort study. BMJ 2012; 344: e1327-e1340.

5. Clark CE, Greaves CJ, Evans $\mathrm{PH}$, et al. Inter-arm blood pressure difference in type 2 diabetes: a barrier to effective management? Br J Gen Pract 2009; 59: 428-432.

6. Clark CE, Taylor RS, Shore AC, et al. Association of a difference in systolic blood pressure between arms with vascular disease and mortality: a systematic review and meta-analysis. Lancet 2012; 379: 905-914. 
7. Banks MJ, Erb N, George P, et al. Hypertension is not a disease of the left arm: a difficult diagnosis of hypertension in Takayasu's arteritis. J Hum Hypertens 2001; 15 : 573-575.

8. Aboyans V, Criqui MH, McDermott MM, et al. The vital prognosis of subclavian stenosis. J Am Coll Cardiol 2007; 49: $1540-1545$.

9. Chobanian AV, Bakris GL, Black HR, et al. Seventh Report of the Joint National Committee on Prevention, Detection, Evaluation, and Treatment of High Blood Pressure. Hypertension 2003; 42: 1206-1252.

10. National Institute for Health and Car Excellence. Hypertension. London: NICE. Available at: http:// guidance.nice.org.uk/CG127 (2011, consulted May 2012).

11. Clark CE, Campbell JL and Powell RJ. The interarm blood pressure difference as predictor of cardiovascular events in patients with hypertension in primary care: cohort study. J Human Hypertension 2007; 21: 633-638.

12. Wilson PWF, D'Agostino RB, Levy D, et al. Prediction of coronary heart disease using risk factor categories. Circulation 1998; 97: 1837-1847.

13. Boehmer TKC, Flanders WD, McGeehin MA, et al. Postservice mortality in Vietnam veterans: 30-year follow-up. Arch Intern Med 2004; 164: 1908-1916.

14. Centers of Disease Control and Prevention. Health status of Vietnam veterans: volume III, medical examination, chapter 9. Atlanta: CDC. Available at: www.cdc.gov/ nceh/veterans/pdfs/VolumeIII/MedicalExamination4_7. pdf (1989, accessed May 2012).

15. Detection, Expert Panel On: Evaluation and treatment of high blood cholesterol in adults. Executive summary of the third report of The National Cholesterol Education Program (NCEP) Expert panel on detection, evaluation, and treatment of high blood cholesterol in adults (Adult Treatment Panel III). JAMA 2001, 285(19): 2486-2497.

16. Consultation WHO. Definition, diagnosis and classification of diabetes mellitus and its complications. Diagnosis and classification of diabetes mellitus 1999; 99(2).

17. Gabir MM, Hanson RL, Dabelea D, et al. The 1997 American Diabetes Association and 1999 World Health Organization criteria for hyperglycemia in the diagnosis and prediction of diabetes. Diabetes Care 2000; 23: 1108-1112.

18. Fowkes FGR, Murray GD, Butcher I, et al. Ankle brachial index combined with Framingham Risk Score to predict cardiovascular events and mortality: a meta-analysis. JAMA 2008; 300: 197-208.

19. Boyle CA, Decoufle P, Delaney RJ, et al. Postservice mortality among Vietnam veterans. Atlanta: US Department of Health and Human Services, Public Health Service, Centers for Disease Control, Center for Environmental Health. Available at: www.getcited.org/ pub/102674747 (1987, consulted May 2013).

20. Kivimäki M, Batty GD, Hamer $M$, et al. Using additional information on working hours to predict coronary heart disease. Ann Intern Med 2011; 154: 457-463.

21. Dudina A, Cooney MT, Bacquer DD, et al. Relationships between body mass index, cardiovascular mortality, and risk factors: a report from the SCORE investigators. Eur J Cardiovasc Prev Rehabil 2011; 18: 731-742.

22. Holme I and Tonstad S. Association of coronary heart disease mortality with risk factors according to length of follow-up and serum cholesterol level in men: the Oslo Study cohort. Eur J Prev Cardiol 2013; 20: 168-175.

23. Harrell FE, Lee KL and Mark DB. Tutorial in biostatistics multivariable prognostic models: issues in developing models, evaluating assumptions and adequacy, and measuring and reducing errors. Stat Med 1996; 15: 361-387.

24. Bozdogan H. Model selection and Akaike's information criterion (AIC): The general theory and its analytical extensions. Psychometrika 1987; 52: 345-370.

25. Schwarz G. Estimating the dimension of a model. Ann Stat 1978; 6: 461-464.

26. Pencina MJ, D'Agostino RB Sr and Steyerberg EW. Extensions of net reclassification improvement calculations to measure usefulness of new biomarkers. Stat Med 2011; 30: 11-21.

27. Chen S-C, Chang J-M, Tsai Y-C, et al. Association of interleg BP difference with overall and cardiovascular mortality in hemodialysis. Clin J Am Soc Nephrol 2012 (Epub ahead of print).

28. Clark CE and Powell RJ. The differential blood pressure sign in general practice: prevalence and prognostic value. Fam Pract 2002; 19: 439-441.

29. Orme S, Ralph SG, Birchall A, et al. The normal range for inter-arm differences in blood pressure. Age Ageing 1999; 28: 537-542. 